\title{
VERS UN PARADIGME JURIDIQUE DE LA TRANSITION RESISTANCIELLE: UNE VISION CONTEMPORAINE DE LA CONTRAPOSITION GEMEINSCHAFT / GESELLSCHAFT DE LA RESISTANCE ITALIENNE A L'OLP
}

\author{
RUMO A UM PARADIGMA LEGAL DA TRANSIÇÃO RESISTENCIAL: UMA VISÃO \\ CONTEMPORÂNEA DA CONTRAPOSIÇÃO GEMEINSCHAFT / GESELLSCHAFT DA \\ RESISTENNCIA ITALIANA À OLP
}

\begin{abstract}
Alessia Magliacane
Doutora em Droit et Sciences Sociales pela Ecole des Hautes Études en Sciences Sociales de Paris; Mestre em Sociologia pela Università degli Studi Suor Orsola Benincasa; Mestre em Droit public comparé européen pela Université de Paris I, Panthéon-Sorbonne; Graduada em Direito pela Università degli studi di Napoli Federico II; Pesquisadora do Centre Georg Simmel da Ecole des Hautes Etudes en Sciences Sociales de Paris; Professora Visitante na Universidade Federal do Rio Grande do Sul (UFRGS).

E-mail: alessiamagliacane@gmail.com
\end{abstract}

Convidada

Je veux parler de vous, les frères de la première heure, les frères en utopie. D'ASTIER DE LA VIGERIE, Avant que le rideau ne tombe [1945].

\section{LES CONCEPTIONS ET LES DEFINITIONS DE «RESISTANCE » DANS LES DIFFERENTS DOMAINES DES SCIENCES HUMAINES ET SOCIALES}

La Résistance est une catégorie d'analyse dans des domaines différents, sans considérer ici les sciences de la nature (qui, d'ailleurs, pourraient nous donner des suggestions intéressantes !). Notamment, l'historiographie, les sciences politiques, la philosophie politique, nous offrent des repères pour comprendre la catégorie de Résistance et pour en saisir la spécificité dans le domaine du droit.

\subsection{La conception historiographique de la Résistance}

L'historiographie définit la Résistance comme une sorte de barrière érigée contre une force visant à détruire, réduire, comprimer les droits garantis par la constitution. ${ }^{1}$

\footnotetext{
${ }^{1}$ Pour une discussion des différents aspects politiques et juridiques que les conceptions de résistance présentent voir le deuxième volume de notre travail de thèse de doctorat portant sur Transition constitutionnelle et Résistance : MAGLIACANE, Le temps non linéaire de la Résistance. A l'épreuve de l'Histoire [2016].
} 
Cette force pourrait être exercée par l'État lui-même vers et contre les citoyens qui n'acceptent pas des mesures ou des politiques qu'ils considèrent anticonstitutionnelles. Pour Boris MirkineGuetzévitch (ce qui lui a par ailleurs été reproché à plusieurs reprises), et dans une vision «jacobine » de la politique, la Résistance et la Constitution expriment toutes deux la tension vers la concrétisation de la démocratie. ${ }^{2}$

Naturellement, si c'est l'État qui « résiste » contre la Nation, donc si c'est une partie du Peuple qui se révolte ou s'insurge contre une autre partie, le concept de Résistance est bien rapproché de celui de guerre civile. ${ }^{3}$

Et pourtant, dans ce cas, on pourrait avoir tout autant deux Résistances qui s'affrontent (alors qu'il est aussi ridicule de parler d'une résistance « fasciste » contre la Résistance partisane, ou d'une résistance « nazie » contre la Libération de Paris !), sans que l'une l'emporte sur l'autre ; qu'une seule catégorie générale de Résistance, dont profitent les deux (ou plus) parties en conflit, jusqu'à ce que l'une d'elles gagne au détriment des autres la légitimité de pouvoir se qualifier de « résistante ». ${ }^{4}$

Pour une vision globale de la Résistance en droit constitutionnel et dans les domaines historiographiques, voir pour l'instant PAVONE, Una guerra civile. Saggio storico sulla moralità nella Resistenza [1991,2009] et le recueil MiCHEL - MiRKINE-GUETZÉVITCH, Les idées politiques et sociales de la Résistance [1954].

Pour un encadrement constitutionnel de la Résistance, avec des observations intéressantes sur le fédéralisme européen et les projets politiques nord-américains, voir HEYDE, De l'esprit de la Résistance jusqu'à l'idée de l'Europe [2010].

${ }^{2}$ Georges Vedel, avec toute la virulence grossière dont il était toujours capable, accusait le grand étudiant de Kiev de vouloir présenter une « démocratie jacobine, qui est la dictature d'une minorité en état de grâce politique sur une majorité en état de péché mortel ... à revenir au droit divin». (Ainsi dans sa Note bibliographique de Les constitutions européennes, de Mirkine, parue sur la «Revue française de science politique », 1952, vol. 4).

${ }^{3}$ Cependant, même sous cette catégorie on peut comprendre et regrouper des phénomènes historiques et politiques, et des concepts d'interprétation, tout à fait différents entre eux et variés. En Italie, la Résistance comme « guerre civile » esquissée par l'historien Claudio Pavone est bien distanciée de la reconstruction historique-politique développée par l'historien du fascisme Renzo de Felice. Ce dernier a été, souvent de façon incorrecte (car il s'agit d'une reconstruction monumentale, la sienne, qui se voudrait politiquement «neutre»), considéré un vulgaire révisionniste, alors que Pavone (qui a forgé l'équivalence dès les années 1980 !) est un historien renommé et accueilli sans problème aucun dans les rangs antifascistes.

Voir donc les textes fondateurs du débat: PAVone, Una guerra civile. Saggio storico sulla moralità nella Resistenza italiana [1991, 2009] et De FeLICE, Mussolini l'alleato [1990 ; 1997] ; DE FELICE, Il rosso e il nero [1995].

${ }^{4}$ Les « deux Résistances » ont marqué l'histoire de la libération de la France, par exemple.

Une vision historique 'simplifiée' se contenterait de voir la lutte pour la légitimation gagnée par la faction politique ralliée à De Gaulle et aux Anglais, mais la IVe République rompt sans ambages avec cette prétendue légitimité gaullienne et gaulliste et, au-delà des erreurs tactiques compréhensibles et de la vision politique autodestructrice qui sont toujours, toutes deux, propres aux communistes lorsqu'ils gagnent du " pouvoir », c'est le Parti Communiste dans un gouvernement de gauche qui doit jouer sa légitimité tirée de la Résistance, et rompre par exemple avec le colonialisme brutal et la continuité impérialiste de la IIIe République. Ce que, par ailleurs, il ne fera pas.

La composition de compromis des gouvernements de transition et des premiers de la IVe République, ainsi qu'une constitution écrite ayant une nature 'ambivalente' - nationaliste et européiste, progressiste dans les droits citoyens, solidariste quant à la fonction de l'État vers la société, modérée en politique étrangère, agressive vers les territoires coloniaux à exploiter, dangereusement décisionniste dans les rapports entre les organes constitutionnels, mais incertaine sur la centralité décisionnelle des organes suprêmes - dériveraient donc de la Résistance concrètement conduite. Une Résistance qui était comme partagée et divisée : une guerre de libération (Résistance des Forces gaullistes), une lutte pour la libération des masses exploitées (Résistance communiste, des formations socialistes en France métropolitaine, du Conseil National jusqu'à l'arrestation de Jean Moulin), une lutte contre l'envahisseur et l'occupant (tous, plus les maquisards), une lutte pour la démocratie à venir (Combat et autres formations indépendantes). 
Les configurations que nous venons de présenter rendent donc plutôt flou l'objet spécifique de la Résistance, qui est ontologiquement renvoyé à l'objet général de la Résistance, que ce soit la libération, la guerre à l'occupant, la forme républicaine ou la lutte des classes.

La Résistance, donc, sous un angle historique et politique n'a pas de limites d'objet ni de dimension. Elle est simplement concrétisée en tout acte susceptible de s'adresser contre la force opprimante..$^{5}$

Les avantages politiques, ainsi que dans la même mesure les limites scientifiques, de cette conception de la Résistance consistent notamment dans la grande versatilité de la catégorie si représentée, qui est donc compatible avec des visions idéologiques différentes et des interprétations controversées de l'histoire.

On parle par exemple de la résistance des Afghans suite à l'invasion militaire de l'OTAN de 2001, de la résistance des armées irakiennes suite à l'invasion de l'OTAN de 2003, de la résistance des loyalistes khadafistes suite à l'insurrection des armées rebelles en Lybie de 2011 . $^{6}$ On parle, depuis longtemps (au moins à partir de 1'exil de 1948), de la résistance palestinienne ${ }^{7}$, et pourtant on a parlé de la résistance israélienne à l'agression arabe pendant la guerre des six jours.

On a parlé de résistance anticommuniste pour légitimer l'existence d'une (ou plus d'une) structure insurrectionnelle clandestine italienne de 1949 à 1990, armée par les EUA et trainée par les services secrets nord-américains et britanniques, nommée Gladio. ${ }^{9}$

Les « deux résistances » sont donc bien, sur le plan historique, politique et constitutionnel, une résistance que nous pourrons définir de continuité et une résistance que nous nommerons par contre de rupture.

${ }^{5}$ Voir, De Felice, Mussolini l'alleato [1990 ; 1997] ; DE FELICE, Il rosso e il nero [1995]. Cette définition englobe par conséquent tant la Résistance en France occupée que celle en France Libre, à Londres ou dans les territoires français outre-mer, et puis encore à Alger à partir de novembre 1942.

Pour la France métropolitaine et la Résistance intérieure prise dans son ensemble, voir DREYFUS, Histoire de la Résistance [1996] qui met bien en évidence la Résistance des Mouvements, notamment des premiers petits groupes (1940-1941) de tendance démocrate chrétienne ou libérale, de toute manière anti-communiste - ce bien avant l'unification et la constitution du Mouvement uni de la Résistance (MUR) - et celle du Conseil national de la Résistance (CNR), organisme où les partis de gauche, en particulier le PCF, tiendront une place déterminante.

Pour la composante syndicaliste-chrétienne de la Résistance en France métropolitaine, voir la belle reconstruction consacrée à l'histoire du mouvement « Libération-Nord » et de Libération, dans ANGLAN, La Résistance sacrifiée [1999].

${ }^{6}$ Afin d'échapper aux difficultés de l'interprétation et au risque d'inexactitude, plusieurs organes d'information européens ont adopté la formule plus romantique de «printemps arabe », ainsi que celle, typiquement millénariste, de « réveil arabe ». Pour une discussion critique, voir ANDRIEU, La justice transitionnelle [2012 : chapitre XVII].

La Résistance italienne avait, elle aussi, connu de telles visions romantiques : voir, par exemple, la vision symptomatique de l'un des chefs des formations du Parti d'Action appelées « Giustizia e libertà », Nuto Revelli, dans REVELLI, La guerra dei vinti [1962]. On peut consulter aussi le beau livre que l'historien italien Sergio Luzzatto a consacré à l'expérience militante résistante de Primo Levi dans son LuZZATTO, Partigia. Una storia della Resistenza [2013].

${ }^{7}$ Un très bon article de l'époque est consacré à la Résistance comme « nouvelle réalité dans les relations israélojordaniennes » par Abdelwahab Hechiche dans le numéro 5 de la revue Politique étrangère de l'année 1973 : HECHICHE, Renaissance et déclin de la Résistance palestinienne [1973]. Voir aussi, toujours les publications de l'époque, DE NoYAnd, Al Fath parle : les Palestiniens contre Israël [1970], CHALIAND, La Résistance palestinienne [1970] et La Résistance palestinienne entre Israël et les Pays arabes [1969].

${ }^{8}$ HECHICHE, Renaissance et déclin de la Résistance palestinienne [1973 : 599].

${ }^{9}$ Hobsbawm parle de «noyaux de résistance » des forces de l'Axe, désormais battues, qui se réunirent dans des structures militaires clandestines soutenues par le gouvernement de EUE. Voir HoBSBAWM, Age of extremes [1994 : 199].

Sauf exceptions, la problématique du niveau proto-insurrectionnel dit «stay-behind», actif tout au long de la période 1948 - 1992, est malheureusement sous-estimée par les historiens. Sur les racines «transitionnelles » de la 
On a parlé d'une seule «Résistance polonaise » qui se révoltait contre les Allemands, d'abord, contre les Anglais, ensuite, et contre les Soviétiques, enfin. De plus, «Résistance polonaise » était également celle des grèves de masse des années 1980 et de l'insurrection contre le gouvernement socialiste.

On relie naturellement la résistance et le « devoir sacré » de défendre la patrie (voir la Constitution italienne, à l'article 52), ainsi que les actes de résistance et la lutte anticoloniale. ${ }^{10}$

Dans le cas de l'anticolonialisme il faut dire brièvement dès maintenant que les positions légalistes (réformistes, fédéralistes, unionistes, conventionnalistes) et les positions indépendantistes (nationalistes, sécessionnistes, africanistes, socialistes, révolutionnaires) de la Résistance anticoloniale, se mélangeaient sur la base d'une même légitimation a contrariis, car la doctrine majoritaire à l'époque était celle de la « communauté organique » (reconnue par exemple au titre XII de la Constitution française de 1958). Si cette communauté présentait des éventuelles distorsions de fonctionnement (ce qui arrivait régulièrement, par ailleurs) les peuples assujettis (ou bien « autonomes » d'après la Constitution !) avaient le devoir constitutionnel de se révolter (contre l'Union, si c'était le cas de la France, notamment). ${ }^{11}$

Ensuite, l'indétermination de l'objet des actes de résistance ${ }^{12}$ rend dramatiquement éclectique la catégorie. Dans le cas célèbre de la Résistance italienne, ce n'est que depuis 2008, en vertu d'une décision jurisprudentielle, qu'on peut parler de « légitimité des actes d'insurrection »,

structure Gladio, dans le sens d'une continuité directe d'avec la structure Osoppo - Terzo Corpo di volontari, créée en 1946 de la section «Clardini » des services secrets du tout nouveau gouvernement du Sud Badoglio, en novembre 1943, et utilisée, après la libération, en clé anti-communiste, PACINI, Le altre Gladio. La lotta segreta anticomunista in Italia. 1943-1991 [2014].

Une curiosité. Dans l'Italie des années 1990 et des premières années 2000, le dirigeant du parquet de Milan, pressé par les attaques des politiques de la droite qui se proposaient de ralentir et de boycotter les investigations judiciaires contre leur leader (Silvio Berlusconi), lançait le slogan «Resistere, resistere, resistere » en s'appelant ouvertement à la saison de la Résistance milanaise et du Risorgimento. Voici l'extrait de son allocution inaugurale de l'année judiciaire 2002, où l'ancien procureur général relie l'abus du droit par le gouvernement et le conséquent «naufrage de la conscience civile», la «moralité » de la lutte judiciaire pour rétablir «le sens du droit», et le «devoir» de Résistance : «Nessuna istituzione, lo so bene, nessun principio, nessuna regola sfugge ai condizionamenti storici e dunque all'obsolescenza, nessun cambiamento deve suscitare scandalo. Purché sia assistito dalla razionalità e purché il diritto, inteso come categoria del pensiero e dell'azione, non subisca sopraffazione dagli interessi. Ma ai guasti di un pericoloso sgretolamento della volontà generale, al naufragio della coscienza civica nella perdita del senso del diritto, ultimo, estremo baluardo della questione morale, è dovere della collettività "resistere, resistere, resistere" come su un'irrinunciabile linea del Piave. »

${ }^{10} \mathrm{Il}$ y a un bon article de l'époque, centré sur les transformations du droit constitutionnel en droit conventionnel (qui était une façon de traduire dans des termes juridiquement acceptables l'anticolonialisme et l'indépendance acquise) qui concerne les destins de la Communauté française-impériale des États africains : BoreLLA, L'évolution de la Communauté en 1960. De la Communauté constitutionnelle à la Communauté conventionnelle [1960].

${ }^{11}$ Toujours pour saisir l'esprit de l'époque, ainsi que les difficultés auxquelles l'anticolonialisme était obligé (par une culture juridique et constitutionnelle opiniâtrement réactionnaire et arriérée) de se confronter : BoRELLA, L'évolution politique et juridique de l'Union française depuis 1946 [1958].

${ }_{12}$ Voir, avec des observations bien claires, CHOMSKY, Rogue States. The rule of Force in World Affairs [2000] ; SAÏD, L'Orientalisme [1978, 2003], Culture et Résistance [2003, 2004], D'Oslo à l'Irak [2004], ainsi que CHOMSKY - SAIID, La loi du plus fort [2002]. En général sur les définitions, en nous limitant à l'après-guerre et aux ouvrages qui contiennent une définition expresse des catégories historiographiques, voir les ouvrages collectifs coordonnés par RANZATO, Guerre fratricide [1994] et par LEGNANI - VENDRAMINI, Guerra, guerra di liberazione, guerra civile [1990], ainsi que PAVONE, La seconda guerra mondiale : una guerra civile europea ? [1994], CRAINZ, Il conflitto e la memoria [1992], LEGNANI, A proposito di storia, stampa e pubblico [1992], VACCARINO, La Resistenza [1990], BARRACLOUGH, Guida alla storia contemporanea [1972 : 28]. Bien différente est la question de la guerre civile en tant que guerre contre les civils : ANDRAE, La Wermacht in Italia [1997], BATTINI - PEZZINO, Guerra ai civili [1997]. 
alors que, au lendemain de l'armistice du 8 septembre 1943 entre le gouvernement Badoglio et les forces alliées, les actes d'insurrection étaient sanctionnés et punis par la loi martiale !

Encore, le manque d'un gouvernement identifié comme légitime pendant la période de la transition (résistance, guerre civile, insurrection, guerre de libération, golpe, putch, etc.) complique et la question et l'interprétation. ${ }^{13}$

Enfin, les repères historiques autorisent souvent à parler, indifféremment, de Résistance, de guerre civile, de guerre de libération, d'insurrection populaire, de coup d'État, selon les données considérées comme les plus importantes.

A savoir que si l'on considère la durée de la transition, on parlera probablement de coup d'État (dans le cas d'une révolution brève) ou de guerre de libération (dans le cas d'une longue révolution avec des fortunes alternées $)^{14}$.

Si l'on se concentre, en revanche, sur les sujets de la transition historique, on parlera de guerre civile (dans le cas d'une Résistance qui se joue sur le même territoire, avec des intérêts en conflit entre eux), de guerre de libération (cette fois-là contre une puissance étrangère qui éventuellement aurait envahi le territoire), de guerre partisane (où la neutralité du terme est compensée par l'unicité des expériences historiques de référence : 1'Italie et la Yougoslavie) ${ }^{15}$, d'insurrection (contre le gouvernement légitime mais... délégitimé).

Nous ne considérerons pas d'autres concepts forgés par les historiens qui parfois compliquent davantage l'interprétation (ceux de rébellion, de révolte, d'agression, de rétorsion, de

13 Voir l'interprétation controversée donnée à la révolution (en fait, la double révolution) antimonarchique iranienne par Michel Foucault, en tant que correspondant du prestigieux quotidien italien Corriere della sera («Lo scià ha cento anni di ritardo », du $1^{\text {er }}$ octobre 1978, p. 1, et « Téhéran : la fede contro lo scià », du 8 octobre 1978 , p. 11, maintenant dans FoUCAULT, Dits et écrits II [1976-1988: 679-683), ainsi que dans A quoi rêvent les Iraniens?, in Le Nouvel Observateur, du 16-22 octobre 1978, 48-49.

${ }^{14}$ Voir, par exemple, au sujet de la Résistance italienne, DONDI, La lunga liberazione [2004]. Il faut souligner que la durée de la transition offre des alternatives conceptuelles qui méritent d'être signalées même si nous n'en partageons pas l'approche conceptuelle et méthodologique. A savoir que d'après les travaux des historiens Rials et Bluche, Emmanuel Cartier donne la définition selon laquelle « au sens politique, la révolution correspond au phénomène de la prise du pouvoir par l'organe révolutionnaire, caractérisé par sa rapidité et sa concrétisation par un fait ou un événement précis qui peut être daté et dont il est possible d'analyser les causes, économiques, sociales, historiques, internes et externes. Au sens de la sociologie du droit ce processus est caractérisé, au contraire, par sa lenteur, son caractère diffus et une origine antérieure à la révolution elle-même ». Ainsi CARTIER, La transition constitutionnelle en France (1940-1945) [2005: 12-13]. Voir aussi RIALS - BLUCHE (dir.), Les révolutions françaises [1989].

${ }^{15}$ En fait, le journaliste-historien italien Giorgio Bocca avait l'intention de donner une connotation factuelle à son analyse de la Résistance, qui reste d'ailleurs parmi les plus significatives et les mieux documentées dans le panorama italien. Il parlait donc d'une «Italie partisane », ainsi que d'une « République partisane », en reprenant les mots des dirigeants de la Résistance italienne (dans laquelle il militait au sein d'une importante formation piémontaise de Giustizia e libertà liée au Parti d'Action et sous la direction stratégique et militaire du Parti Communiste). Il reste, malheureusement, que Bocca n'a pas défini expressément l'autonomie de la guerre partisane par rapport à la guerre antifasciste (avec laquelle, par contre, il l'identifie souvent). Voir BocCA, Storia dell'Italia partigiana [1995], Partigiani della montagna [1945, 2004], Una Repubblica partigiana [1964].

«Guerre partisane » est une formule présentée historiquement par l'un des chefs de la Résistance italienne, Dante Livio Bianco: BIANCO, Guerra partigiana [1954, 2006]. Voir aussi CIPRIANI, Guerra partigiana [1945], BRAVO, La repubblica partigiana dell'Alto Monferrato [1964].

En interprétant la nature historique et politique de la guerre « partisane » nazi-fasciste, ainsi que de « république partisane », on a parlé comme en miroir de « république des chemises noires » : voir GANAPINI, La repubblica delle camicie nere [1999]. 
retaliation, d'ouverture des hostilités... ou, pire, de terrorisme, guérilla ${ }^{16}$, etc. $)^{17}$, souvent en détachant incompréhensiblement le fait local du contexte général international. ${ }^{18}$

En conclusion, la catégorie historiographie de Résistance semble parfois ambiguë, confuse, viciée d'éclectisme et donc peu utile pour la comparaison juridique, ce qui, incidemment, a souvent déterminé la sous-estimation de la catégorie de Résistance en général au sein des études juridiques.

\subsection{La conception de la Résistance dans les domaines des sciences politiques et de la politologie}

Installée en position ancillaire notamment par rapport à la Révolution et à la guerre civile, la Résistance a néanmoins connu un bref succès en tant que catégorie des sciences politiques, en particulier lorsqu'elle a été appliquée pour expliquer certaines révolutions qui pouvaient se considérer inachevées ${ }^{19}$ ou des prises du pouvoir qui se révélaient fort problématiques. ${ }^{20}$

\footnotetext{
${ }^{16}$ Avec les meilleures intentions, en étant aussi une sorte d'essai-témoignage, LEVI CAVAGLIONE, Guerriglia nei Castelli Romani [1945]. Voir aussi PIETRA, Guerriglia e contro guerriglia. Un bilancio militare della Resistenza [1997].

${ }^{17}$ Qu'il soit clair que nous ne soutenons pas que l'interprétation doive être forcément simple ou aisément compréhensible, mais que les catégories devraient servir à catalyser la complexité nécessaire du fait historique et non pas à la rendre fumeuse ou, pire, à l'asservir à une idéologie. L'exemple de la Résistance italienne est dramatiquement populaire dans la littérature. Un exemple plus complexe et moins connu est celui de l'Espagne des années '30 au sujet des anarchistes et, surtout, des carlistes de la Navarra. Toujours par rapport à l'Espagne, la guerre civile de 1936 est souvent appelée (sans trop de souci) guerre antifasciste ou, plus correctement, guerre républicaine. Voir HoBSBAWM, Age of extremes [1994: 195], qui souligne l'importance acquise par la méthode gradualiste (parlementaire) au détriment de la méthode de l'insurrection (populaire).

${ }^{18}$ Erreur qui n'est pas commise par HoBSBAWM, Age of extremes [1994 : 173].

${ }^{19}$ C'est le cas emblématique de l'Italie du Nord, où le projet de la guerre de classe et de la révolution socialiste, affirmé par le Parti communiste dès ses origines, se rallia progressivement à celui de la « démocratie progressive » en raison du compromis institutionnel dérivant de la composition du gouvernement italien de 1944. En effet la guerre de classe a maintenu ouvertes ses «projections » jusqu'à l'après-guerre (voir les actions militaires de lutte antifasciste dans le «triangle rouge » de l'Italie du Nord-Est) et, par la suite, jusqu'à la constitution de groupes et de mouvements armés pendant les années 1960 (par exemple les Gap de l'éditeur renommé Giangiacomo Feltrinelli), les années 1970 (éminemment les Brigades rouges, qui introduisaient ouvertement le projet de «continuer la résistance », entendue évidemment comme guerre de classe trahie par les «accommodations » du Parti communiste), les années 1980 (notamment le Parti communiste combattant et le Parti de la guérilla, dérivés tous deux de la scission problématique des Brigades rouges).

Une analyse détaillée des révolutions «inachevées » est contenu dans le premier volet de la reconstruction historique de la révolution russe par Léon Trotsky : TROTSKY, Histoire de la révolution russe. 1 : La révolution de février [1929, 1995].

${ }^{20}$ Sous la notion de « résistance » dans un sens flou est, par exemple, comprise et présentée la lutte nationaliste du peuple irakien suite à la prise partielle du pouvoir en 2003 par l'OTAN - il s'agirait en réalité de la «coalition multinationales en Irak (qui serait parfois à considérer légitime en droit international suite à la reconnaissance par la communauté internationale du fait de la destitution du président Saddam Hussein, mais qui ne s'accompagnait pas du contrôle militaire et politique du territoire, qui n’était donc pas légitime en droit constitutionnel).
} 
La Résistance est donc une catégorie résiduelle et éventuelle, qui assume toute importance uniquement par référence au phénomène révolutionnaire, dont elle regroupe les prodromes ${ }^{21}$, les épiphénomènes secondaires ${ }^{22}$, ainsi qu'une série particulière d'effets directs ou indirects ${ }^{23}$.

L'exemple presque paradigmatique de Résistance dans ce domaine est la Résistance palestinienne, servante d'une révolution renvoyée à jamais.

Elle comprend, au moins

a) des actes épars de la part de la population,

b) une organisation embryonnaire de pouvoir politique,

c) une stratégie insurrectionnelle,

d) une proposition constitutionnelle,

e) une série de revendications résiduelles en cas d'échec.

a) Les actes de la population. C'est le premier élément qui rend intéressant le concept de résistance dans le domaine politique : le fait du peuple. La résistance émane du peuple, ce qui la différencie politiquement de la révolution, qui peut bien être organisée par le sommet du pouvoir politique ou qui peut être inspirée par une seule partie du peuple sur une ligne nationaliste, minoritaire, avantgardiste, génériquement antiautoritaire, antimonarchique, antifasciste, etc. ${ }^{24}$

${ }^{21} \mathrm{La}$ Résistance est souvent interprétée comme instrumentale et fonctionnelle à l'insurrection. Elle serait l'avant-garde de la Révolution, alors que l'insurrection en serait l'aboutissement. Dans le cas de la Résistance italienne de 1943-1945 les actes de résistance étaient reconduits sous la finalité plus vaste de l'insurrection générale. De plus, tant le gouvernement du Royaume du Sud que le Comité de libération (à savoir : les deux organismes suprêmes de la Résistance et de la guerre de libération en Italie) se disputaient la primauté pour la déclaration de l'insurrection générale.

22 La composition des gouvernements provisoires et la négociation politique, notamment entre les partis politiques et les organes institutionnels, se perfectionnent au sein du mouvement de la Résistance. C'est évident qu'il ne s'agit pas de phénomènes d'importance secondaire, mais «dérivée », car la Résistance organise les sujets et sélectionne les finalités. Un exemple historique majeur est l'évincement par De Gaulle de ses adversaires : Giraud, le Conseil de la Résistance, les forces communistes combattantes, le Gouvernement provisoire. Jusqu'à centraliser dans ses mains seules la légitimation de la guerre de libération. Eh bien cette centralisation progressive s'acheva au sein du mouvement de la Résistance.

Sur le plan historique, lorsqu'il se produit une centralisation fonctionnante et rapide (tel est le cas de la Résistance française, le premier acte d'insurrection étant la libération de la Corse en octobre 1943, suivi par l'évincement du co-président du Comité de libération nationale en novembre 1943 : un mois après !), les finalités révolutionnaires cèdent à celles de la libération nationale.

${ }^{23}$ Dans ce cas la discussion est plus délicate, activant une série d'évaluations sur la nature des actes de résistance. Si la Résistance débouchait sur une Révolution, les actes, même s'ils sont conduits sous des formes « terroristes », resteraient toujours valides sur le plan juridique, leur contenu violent étant absorbé et légitimé par les finalités et par la stratégie de la guerre de libération. Si, par contre, la Résistance débouchait sur une restauration constitutionnelle, même sous une forme démocratique plus avancée et progressiste, s'ouvrirait un problème d'interprétation, les finalités constitutionnelles nouvelles ne pouvant pas englober les actes de terrorisme et une violence qui apparaitrait comme injustifiée et illégitime.

Cette polarisation s'est produite à maintes reprises : les actes de résistance des partisans italiens à Rome, Milan et Florence, par exemple (célèbre est le cas de l'attentat à une division nazi à Rome, via Rasella, le 24 mars 1944 , suivi par des représailles nazis consistant dans le massacre de 335 prisonniers) ; les actes de « terrorisme » des organisations pour la libération de la Palestine ; la récente guerre de résistance du peuple irakien contre les occupants de l'OTAN ; mais également des actes symboliquement fondamentaux : l'attentat contre Giovanni Gentile perfectionné par le Gap de Florence le 15 avril 1944, ou l'exécution de Mussolini par le Gap de Milan ou par le « commandant Valerio » (probablement Luigi Longo lui-même), selon les versions, le 28 avril 1945.

${ }^{24}$ La résistance ne serait pas admise comme catégorie juridique de la postmodernité ou simplement de l'actualité historique contemporaine par les juristes qui dépriment le concept de « peuple » en tant que correspondant à la figure du pouvoir étatique moderne : NEGRI, Il potere costituente [1992, 2002]. « Il concetto di popolo è concetto falsificante, poiché prodotto per attrazione ed assimilazione dal potere statuale moderno. Popolo è una produzione ideologica

Revista de Direito Brasileira | Florianópolis, SC | v. 22 | n. 9 | p.149-170 |Jan./Abr. 2019 
b) Une organisation du pouvoir. Le caractère démocratique et populaire de la résistance n'en réduit pas la dimension organisationnelle. Une résistance se dote d'une direction de l'activité qui en principe devrait cordonner (ou, au moins, revendiquer) les actes et s'assumer surtout le pouvoir de construction d'une stratégie politique. ${ }^{25}$

c) Une stratégie insurrectionnelle. Il s'agit d'un élément éventuel, qui est présent dans les rébellions les plus organisées, ainsi que dans celles qui se concrétisent en une prise du pouvoir politique (ce qui caractérise proprement une révolution au sens politique du terme). ${ }^{26}$

borghese che si oppone a moltitudine. Finché esistono quel concetto di sovranità e questo concetto di popolo, non siamo ancora nel postmoderno, ovvero nell'epoca che stiamo vivendo ». Voir NEGRI, Il potere costituente [2002 : 10].

${ }^{25} \mathrm{Il}$ est couramment souligné que la différence entre révolution et rébellion est la vision stratégique au niveau politique, qui manquerait aux sujets qui organisent ou conduisent une rébellion. Les exemples en sont les riots urbains de Los Angeles de 1988, les actes de dévastation dans les banlieues parisiennes de 2004 et 2005, ainsi que les mouvements pré-insurrectionnels qui n'aboutissent pas à une révolution achevée (c'était le cas de l'Afrique du Sud, et plus récemment c'est le cas de la Syrie de 2011).

La rébellion peut également revêtir une apparence tactique : notamment les actes de sabotage peuvent être interprétés comme des actions spécifiques et 'ponctuelles' d'une stratégie plus vaste. Le parti de l'ANC en Afrique du Sud s'est engagé et perfectionné dans la pratique du sabotage, en la justifiant comme une tactique qui aurait fini pour défatiguer le gouvernement. Voir MANDELA, Un long chemin vers la liberté [1995]. Voir RIGAULT - SANDOR, Le démantèlement de l'Apartheid [1993], et le bon volume PORTEILLA, Le nouvel État sud-africain. Des Bantoustans aux provinces [1999].

Toujours au sujet de l'Afrique du Sud, il faut mentionner l'observation intéressante du juriste et historien marxiste Martin Légassik qui constate que «la transition sud-africaine n'est pas une transition vers le socialisme et bien des aspects de l'apartheid restent présents ». Il s'agit plutôt d'une " victoire impressionnante de la transition ». Voici donc quant au manque du fait du peuple. «Portée sur les fonts baptismaux au moment même où s'effondrait le socialisme réel, la Nouvelle Afrique du Sud peut difficilement, en dépit de la considération dont elle jouit aujourd'hui dans le monde, apparaître comme un nouveau modèle de société : la croissance qui devait permettre une nouvelle distribution des richesses et une nouvelle forme de développement n'est pas au rendez-vous, les inégalités sociales restent gigantesques, l'emploi a régressé, l'insécurité constitue un problème qui va en s'aggravant. En un sens, l'Afrique du Sud a réintégré la communauté banale des nations, notamment africaines, soumises à la loi d'airain des marchés mondiaux, avec sans doute cette particularité que la démocratisation y a précédé l'ajustement. » Voir LÉGASSIK, Myth and Reality in the Struggle against Apartheid [1998 : 3]. Sur la même ligne MEUNIER - COPANS, Afrique du Sud. Les débats sur la transition : les ambiguïtés de l'ère Mandela [1999].

Voir aussi le reportage paru sur Financial Times du 26 octobre 2015 concernant la vague des protestations montée contre le gouvernement, notamment par les comités universitaires des étudiants black, dans celle qui a été définie comme «the post-apartheid era ». "'The majority of South Africans have been very naive, pushing the Raimbow Nation, whatever you call it', says Majaletje Mathume, a student activist at Stellebosch University. To a great extent, a lot of black South Africans, still feel as if they are subservient to white people. Transformation was predicated on not hurting their (white) feelings, not making them run away ». Il suffit de penser que dans cette prestigieuse université, environs $60 \%$ des étudiants sont blancs, et que la langue dominante utilisée dans les leçons est encore l'Afrikaans. Voir ENGLAND, Barriers to entry [2015 : 5].

${ }^{26}$ La stratégie de l'insurrection est fondamentale afin de pouvoir saisir la différence de catégorie politique entre la résistance et une prise du pouvoir quelconque, cette dernière ne contemplant pas forcément le passage insurrectionnel. De surcroît, on ne peut même pas considérer la résistance comme une espèce de prise du pouvoir, comme si cette dernière était la catégorie générale et l'autre celle plus spécifique : si la résistance ne parvenait pas à la prise du pouvoir ou à l'éversion de l'ordre constitutionnel, on pourrait la qualifier d'une simple « rébellion », sans spécificité ni autonomie comme catégorie politique. Ce qui évidemment n'est pas correct sur le plan historique, car une résistance qui n'est pas aboutie comme prise du pouvoir peut également produire des effets politiques, juridiques, constitutionnels.

L'exemple de l'Organisation pour la libération de la Palestine en est sans doute le plus important et cité, mais il faut considérer aussi le cas de la guerre de classe des partisans communistes en Italie (1943-1947) où la Résistance (en tant que guerre de classe qui n'avait pas réussi la prise du pouvoir) a été le fondement constitutionnel de la nouvelle république postfasciste et post-monarchique instaurée via la constitution républicaine de 1948. 
d) Une stratégie constitutionnelle. Cet élément est également éventuel, et pourtant il est souvent présent dans les rébellions qui revendiquent un ordre politique différent de celui au pouvoir. ${ }^{27} \mathrm{C}$ 'est le cas typique des résistances contre un régime dictatorial, où les idées constitutionnelles de la Résistance conflueront dans la nouvelle constitution.

e) Des revendications résiduelles. Cet élément est presque universellement oublié par les analystes politiques (et juridiques) de la résistance, l'échec de celle-ci étant pourtant le cas historique le plus répandu, diffus et connu. Ce que nous appelons « revendications résiduelles en cas d'échec » sont, en effet, les éléments qui nos autorisent à envisager le cheminement progressif de la construction d'un nouvel ordre politique et normatif. De plus, dans le domaine des sciences politiques (cette fois accompagné par des éléments de science économique), la conduite des groupes révolutionnaires qui ont une importance mineure est souvent liée à la figure du «passager clandestin $»^{28}$.

L'importance de la résistance comme catégorie politique est donc reliée essentiellement à la participation populaire (éventuellement organisée ou assumée par les partis politiques) et à la stratégie de dépassement du régime de pouvoir (éventuellement par la revendication d'une nouvelle constitution).

La Résistance correspond donc à une phase propédeutique, nécessaire et parfois incontournable, du mouvement révolutionnaire (soit-il abouti ou non).

Nous proposons l'hypothèse que la « transition résistancielle » est un paradigme adéquat et complexe afin de saisir la spécificité des transitions constitutionnelles, du moins par rapport à un cycle constitutionnel proche de nous dans le temps: celui de l'entière longue vague du constitutionnalisme de l'après-guerre jusqu'à aujourd'hui.

\section{VERS UN PARADIGME DE LA TRANSITION « RESISTANCIELLE »}

Une transition qui soit «résistancielle » ne serait pas 'seulement' "résistante », cette dernière incarnant essentiellement la nature des luttes ${ }^{29}$ alors que la transition « résistancielle » incarnerait une ligne intermédiaire entre la transition constitutionnelle et celle révolutionnaire.

27 Même les émeutes de Los Angeles de 1988 avaient des propositions que nous pourrions qualifier de « constitutionnelles », au moins dans le sens où ces mouvements spontanés revendiquaient un rôle différent de l'ordre public et des pouvoirs politiques, ainsi qu'une redistribution des richesses et un élargissement de droits civils et de garanties constitutionnelles en faveur des minorités ethniques et des rangs les plus pauvres de la population.

${ }^{28}$ Le grand juriste et philosophe italien Norberto Bobbio a souvent souligné comment les communistes avaient « fait » la Résistance militaire contre les nazi-fascistes en Italie alors que les catholiques avaient joué un simple rôle passif, avec une participation toute symbolique à la guerre de libération, qui consentira pourtant au parti catholique de la DC de gagner une force de revendication paritaire au sein des gouvernements de guerre (dirigées par les communistes), de l'assemblée constituante (toujours présidée par un communiste), du comité dit « de 75 » qui a rédigé la charte constitutionnelle italienne (comité présidé par un communiste), jusqu'à arriver à gagner les premières élections démocratiques et à suffrage universel après l'approbation de la nouvelle Constitution républicaine ! Voir BOBBIO - RUSCONI, Dialogo sull'antifascismo [1992].

En fait, la même observation que Bobbio concentrait sur les communistes italiens pourrait être référée, par exemple, aux communistes iraniens après la révolution islamiste de 1979.

Plus récemment, les kurdes en Syrie ont été chargés de conduire la résistance sur le territoire contre l'insurrection islamiste d'un prétendu « Etat islamique d'orient » (Isis), en échange de l'autonomie fédéraliste de leur territoire.

29 Pour une analyse du glissement de sens que la définition prend vers le phénomène du « terrorisme », qui serait une frontière ultérieure à franchir sur la voie de l'évolution des formes du Politique, notre MAGLIACANE, Rupture de l'Histoire, continuité de la Résistance [2017] ; aussi le plus récent L'uomo Mosè e il moroteismo [2019].

Revista de Direito Brasileira | Florianópolis, SC | v. 22 | n. 9 | p.149-170 |Jan./Abr. 2019 
La transition que nous disons de résistancielle est donc comme tendue et suspendue entre deux débouchés historiques possibles: l'Etat démocratique constitutionnel, et l'Etat révolutionnaire.

Encore, la transition résistancielle s'appuierait sur la complexe phénoménologie de la Nation, plutôt que sur la simplification historique-politique-juridique (qui est surtout inutilisable scientifiquement) du Peuple souverain (qui incarne en revanche le présupposé fondationnaliste ou bien de la Constitution ou bien de la Révolution, et tant de l'une que de l'autre).

Enfin, en raison de ce caractère «suspendu » de la stratégie résistancielle, qui vise parfois la révolution mais qui tombe concrètement sur la constitution, le concept même de Nation se présenterait toujours associé à d'autres concepts : la libération, dans la transition résistancielle, est donc nationale, et les sujets - par exemple les comités de libération - sont des sujets nationaux de libération nationale (nuance qui échappe couramment lorsqu'on les condense sous la formule de CLN). ${ }^{30}$

\subsection{Le cas paradigmatique de l'OLP}

Le paradigme de transition « résistancielle» que nous proposons ici est comparable à la condition de l'OLP depuis sa fondation jusqu'à la constitution de l'Autorité nationale palestinienne suite aux accords d'Oslo (et même après, en vérité). ${ }^{31}$

L'OLP représentait légitimement un peuple et visait à la constitution d'un État. ${ }^{32}$

La condition de l'effectivité de l'oppression est respectée, l'État d'Israël ayant plusieurs fois violé les confins nationaux et agi avec la proposition d'écraser militairement l'OLP, tout en empêchant la constitution de l'État de Palestine (qu'il occupait illégalement).

La condition du normativisme - l'indifférence éthique, morale, idéologique, politique - est elle aussi respectée dans le cas de l'OLP qui, de plus, a un projet de constitution, voire de constitution d'un État. ${ }^{33}$

Dans le même temps, l'OLP n'a pas agi de façon révolutionnaire; de même la stratégie révolutionnaire ne peut être envisagée dans la subversion de l'ordre juridique... de l'État d'Israël (ou, pire, dans un projet générique antisioniste).

Sur le «portrait » historique du terroriste, SCAMARDELLA, Verità e paura [2017].

${ }^{30} \mathrm{La}$ stratégie nationaliste, mais ouvertement patriotique aussi, sinon 'seulement' territoriale, n'est pas incompatible avec une vision élargie, de classe, anti-impérialiste, etc., même si, sur le plan historique, un mouvement qui avait une nature de «lutte de classe», tel le front algérien, s'est souvent appelé "populaire » plutôt que « national », toujours en s'ajoutant la formule « de libération ». C'est vrai, de toute façon, que les mouvements et les fronts ont pris des adjectives variables selon les combinaisons des forces politiques, populaires, nationales, ethniques, engagées et mélangées.

${ }^{31}$ Perry Anderson s'est penché sur le sujet dans son dernier éditorial de la new Left review de 2015, où nous lisons que «The Palestinian Authority established in 1994, presented as a milestone in the struggle for national liberation, was in design a co-production of the West and of Israel, whose primary function was not to embody but to contain resistance to Zionism ». ANDERSON, The House of Zion [2015 : 5].

32 Pour une présentation des questions juridiques, de droit constitutionnel et international, voir GUARINO, Personalità giuridica di diritto internazionale : il caso dell'Organizzazione per la Liberazione della Palestina [2004].

${ }^{33}$ Pour une analyse détaillée du débat interne aux Conseils nationaux palestiniens sur " l'Etat démocratique » et de l'évolution de la pensée politique de la Résistance palestinienne, voir l'étude classique de GRESH, Olp. Histoire et stratégies. Vers l'Etat palestinien [1983].

Alain Gresh est revenu sur la «question palestinienne » dans un livre-mémoire qui essaie de la situer dans le nouveau contexte international et de la délier d'une «question juive» dans GRESH, De quoi la Palestine est-elle le nom? [2010]. 
Il s'agit, donc, d'une transition que nous définissons de « résistancielle », comparable à celle de la Résistance italienne contre l'oppression nazi-fasciste (en disant occupation, nous cacherions la matrice fasciste et nationale de l'oppression). ${ }^{34}$

\section{PEUPLES, NATIONS, ETHNIES, MINORITES}

Quelques historiens ont publié des ouvrages, mais ils n'ont retenu que les faits et gestes de quelques personnages. Ils ne semblent pas avoir aperçu le principal acteur du drame : le peuple. TOLLET, La Classe ouvrière dans la Résistance [1984, 1969 : 11].

Ce qui se passe en Iran a de quoi troubler les observateurs d'aujourd'hui. Ils ne peuvent y retrouver ni la Chine, ni Cuba, ni le Vietnam, mais un raz de marée sans appareil militaire, sans avant-garde, sans parti. Ils n'y retrouvent pas non plus les mouvements de 1968, car ces hommes et ces femmes qui manifestent avec des banderoles et des fleurs ont un but politique immédiat; ils s'en prennent au chah et à son régime; et ils sont bel et bien ces jours-ci en train de les renverser. FOUCAULT, Une révolte à mains nues [1978, 2001 : 701]

Les problèmes de définitions sont toujours à l'œuvre. Dans le cas de la Résistance nous pouvons arriver à identifier jusqu'à quatre acteurs différents reconnus par le droit international et par le droit constitutionnel, parfois avec des nuances sémiologiques et des glissements de sens politique qui affectent tantôt la définition elle-même tantôt le concept.

Il n'y a pas lieu ici de parcourir les sentiers théoriques, méthodologiques et politiques qui ont caractérisé les concepts de peuple, nation, ethnie et minorité tout au long de l'évolution de la science juridique contemporaine (d'autant que le faire en référence à l'histoire entière du droit, même jalonnée par étapes fondamentales, serait proprement impossible). ${ }^{35}$

Ce que nous trouvons fructueux dans le cadre de cette recherche, c'est de présenter quelques aspects problématiques des notions considérées, surtout en ce qui concerne les relations complexes de tels concepts entre eux, et de proposer des solutions aux contradictions qui ont souvent accompagné les exposées juridiques.

34 Dans des termes qui soient juridiquement corrects, nous pouvons parler de guerre de libération en Italie uniquement en référence à la période successive à la constitution de la République sociale italienne (octobre 1943), et aux territoires qui ne rentraient pas dans les confins de ladite RSI.

Il y a, donc, une Résistance italienne :

a) pendant les années 1943-44 sur les territoires qui feront successivement partie de la RSI et jusqu'à sa constitution,

b) pendant les années 1943-1945 sur les territoires qui ne font pas partie de la RSI, puisqu'il y a Résistance

c) à Rome capitale, pendant la période de la «ville ouverte »,

d) et également au Sud de l'Italie au lendemain de l'armistice du 8 septembre 1943, juin 1948).

e) en Italie au lendemain de la libération du 24 avril 1945 et jusqu'à l'entrée en vigueur de la Constitution (2

Sur les points $s u b$ a), b), c), d), on peut consulter SCOPPOLA, 25 aprile. Liberazione [1995].

${ }^{35}$ Ainsi, nous ne pouvons pas nous pencher dans le cadre de ce travail sur la Catalogne et le cas de la sécession. Pour une discussion, nous renvoyons aux travaux de la constitutionnaliste et commentatrice politique espagnole Teresa Freixes : voir, entre autres, le plus récent FREIXES, 155 : Los días que estremecieron a Cataluña [2018]. 
Pour commencer, nous noterons qu'il y a une certaine confusion, chez les constitutionnalistes notamment, autour des gradations de «valeur» entre peuple et nation. Voici des gradations particulièrement intéressantes aux fins de cette recherche.

a. Le peuple est l'entité qui fonde l'Etat de droit, alors que la nation en est l'une parmi plusieurs (ou, au moins, plus d'une) caractérisations sociologiques. Il a été inféré que «Peuple » correspond à Etat, tandis que « Nation » correspond à Société ${ }^{36}$ dans une sorte d'opposition tantôt épistémologique ${ }^{37}$ tantôt constitutionnelle ${ }^{38}$. Soulignons que, dans le cadre d'une telle proposition interprétative, l'ethnie ou la minorité (ethnique, linguistique, politique, religieuse, etc. $)^{39}$ ne sont que spécifications sociologiques de la nation. ${ }^{40}$

b. Or, contre cette vision « gradualiste » qui est pourtant majoritaire et souvent acceptée de manière a-problématique, nous devons pourtant dénoncer que l'histoire récente et le droit international nous confient des cas de relation stricte entre Nation et Etat, tout en reléguant l'importance du peuple à un niveau essentiellement politique et revendicatif. Ajoutons encore que, si cette importance politique du peuple devait parvenir à une considération juridique-constitutionnelle, elle serait sans doute problématique. Prenons le cas de la Palestine, ou mieux des territoires occupés par l'armée israélienne dès $1948^{41}$. Le concept de peuple groupait et les Palestiniens qui habitaient les territoires depuis toujours, et les Israéliens qui arrivaient (ou revenaient, selon eux !) légitimés par la célèbre et ambiguë résolution de la Société des Nations portant sur le «national home» des juifs. Le mouvement historique qui fonde l'Etat israélien est donc la nation israélienne, alors que le mouvement historique qui aurait le droit, le pouvoir, fondateur d'un Etat palestinien est la nation palestinienne. ${ }^{42}$

c. L'exemple de la Palestine nous amène à évaluer l'hypothèse que et le peuple et la nation aient une sorte de pouvoir constituant. ${ }^{43}$

36 Voir GRESH, De quoi la Palestine est-elle le nom ? [2010 : 27, 29].

${ }^{37}$ Peuple et Nation comprennent des objets différents.

${ }^{38}$ La souveraineté est, par exemple, une condition du peuple et non pas de la nation. En droit international, par contre, la souveraineté est une condition des nations. Pour les unions supranationales, enfin, la souveraineté est une condition des Etats qui se dialectise (problématiquement) avec la souveraineté des Peuples prévue dans les constitutions.

39 Une implication intéressante concernerait la Nation, l'Ethnie et la Ville, suivant la ligne analytique intelligente de George Simmel : voir, par exemple, PAQUOT, L'invention du citadin [1997] ou MonGIN, Vers la troisième ville? [1995]. [1985].

Pour une interprétation wébérienne voir, par exemple, RÉMY, La ville dans la problématique wébérienne

${ }^{40}$ A peu d'exceptions près, notamment pour ce qui concerne les minorités politiques - ce qui est, par ailleurs, problématique dans le cas où une minorité prendrait le pouvoir, tout comme en Afghanistan suite à l'intervention de représailles de l'OTAN de 2001, ou, plus clairement, en Irak suite à la guerre « unilatérale » contre le pays de 2003 et à la chute du gouvernement légitime - nombre de constitutionnalistes s'accordent sur ce caractère dérivatif et dérivé du concept d'ethnie.

${ }^{41}$ En employant le terme « armée » nous n'entendons pas légitimer politiquement la présence des Israéliens comme si elle émanait d'un pouvoir étatique doté, assisté, de la force militaire. En fait, il s'agit d'une occupation militaire illégitime et illégale.

${ }^{42}$ Ce qui, à notre avis, permet d'envisager, parmi d'autres crimes et violations, la responsabilité israélienne de génocide envers et contre le peuple de la Palestine, qui n'existe plus depuis l'occupation militaire et productive (les occupations militaires et productives) des territoires palestiniens.

${ }^{43}$ Ecartons pour l'instant d'autres « entités » telles, notamment, la communauté, la multitude, la commune, le commun. Même si les auteurs qui les proposent donnent à ces formations un pouvoir constituant (bien entendu, de différente importance), nous estimons que l'originalité de la communauté est plus basse que celle de la nation (et donc limite davantage le caractère d'essentialité que cette première incarne); par contre la connotation politique et 
d. Et que ce pouvoir constituant soit partagé par le Peuple et la Nation.

\subsection{Les mouvements de libération et les gouvernements provisoires}

La problématique que nous venons d'esquisser brièvement est en effet contradictoire, car elle postule en faveur d'une 'compétition' pour le pouvoir constituant (qui serait spéculaire à la lutte pour la résistance), mais dans le même temps suppose aussi que l'objet du pouvoir constituant (et, symétriquement, de la résistance) soit identique pour toutes les parties qui le revendiquent (ou en revendiquent une relation quelconque, de nature variable, entre la titularité constitutionnelle et l'exercice politique).

Il y a plus. Car la forme du Pouvoir constituant (et de la Résistance) s'impose comme une configuration de compromis entre la lutte pour le pouvoir constitué incarnée par la transition constitutionnelle et celle pour le dépassement du pouvoir constituant incarnée par la transition révolutionnaire.

Quant aux sujets des luttes, il y a bien évidemment au moins deux aspects fondamentaux qui caractérisent l'encadrement des mouvements de libération et des gouvernements provisoires : d'abord un aspect passif-agressif, à savoir le devoir patriotique, nationaliste, solidaire, de la défense du territoire ; ensuite un aspect que nous appellerons ab-réactif, à savoir la centralisation active de la force.

\subsection{La défense de la patrie et les garanties constitutionnelles du droit de résistance}

Le premier aspect fondamental caractérisant l'encadrement des mouvements de libération et des gouvernements provisoires, nous l'avons défini comme un aspect passif-agressif, à savoir le devoir patriotique, nationaliste, solidaire, de la défense du territoire.

Cette passivité, avec la dimension seulement éventuelle de son activation, le différencie par fonction de l'aspect que nous définirons ab-réactif, à savoir la centralisation active de la force.

scientifique-culturelle de la multitude est beaucoup plus vaste que celle qui nous permettrait d'envisager la portée historique de son pouvoir constituant (sauf dans le cas, entièrement hypothétique, du cosmopolitisme, cas qui, par ailleurs ne réduit pas les conflits entre les nations ou les ethnies qui, au moins dans une première phase, constituent la multitude) ; troisièmement, nous notons que la commune est tantôt trop petite dans sa portée constituante (ce qui n'est pas un problème théorique, évidemment, mais qui rend incomparable l'exemple politique de la commune avec les phénomènes historiques des Etats et des communautés d'Etats), tantôt trop avancée pour ce qui est de sa définition historique (à savoir sa vocation insurrectionnelle et sa nature communiste, les deux également incompatibles avec la nature étatique et d'ordonnancement de la nation et du peuple). Parmi les auteurs considérés, Pierre Dardot et Christian Laval dans leur DARDOT - LAVAL, Commun. Essai sur la révolution au XXI siècle [2015], et Antonio Negri dans NEGRI, Inventer le commun des hommes [2010].

Si la multitudo est de dérivation spinozienne, la commune est de dérivation leibnizienne : voir BASSO, Individuo e comunità nella filosofia politica di G. W Leibniz [2006], Inquietudine e politica in Leibniz. [2005], RILEY, Leibniz. and the idea of the common good [2002].

La position d'Ernesto Laclau est remarquable quant à la présupposition d'un peuple qui serait déjà hégémonique par rapport à l'Etat, ce qui nous rapproche de la vision gramscienne de peuple et surtout du domaine du « populaire » présenté sous une forme presque paradigmatique dans le Quaderno 22 de la prison. Voir GRAMSCI, Americanismo e Fordismo [1978].

Parmi les nombreux auteurs qui ont analysé la position gramscienne, nous suggérons ici la reconstruction historique proposée par SALVADORI, Gramsci e il problema storico della democrazia [1972]. Voir aussi le dense pamphlet de BOBBIO, Gramsci e la concezione della società civile [1975]. En fait le fondateur du Parti Communiste d'Italie avait proposé une formule heuristique et critique très intéressante, celle de « bloc historique ».

Revista de Direito Brasileira | Florianópolis, SC | v. 22 | n. 9 | p.149-170 |Jan./Abr. 2019 
Contrairement à une vision simpliste de la dialectique kelsenienne du pouvoir étatiqueconstitutionnel, le grand constitutionnaliste aux sympathies gauchistes proposait un mouvement double, à la fois centripète et centrifuge, au cours de la formation de l'Etat constitutionnel par rapport à l'appropriation de la violence sociale généralisée, dont le droit constituait une première forme d'encadrement et d'organisation (n'oublions pas, chers lecteurs, une toute première forme, et absolument pas une forme aboutie ou développée).

Penchons-nous brièvement donc sur cette vision kelsenienne aussi bizarre que courageuse de la force qui serait gérée par une « communauté ».

\subsection{La community kelsenienne et l'habilitation au monopole de la force}

L'Iran est actuellement en état de grève politique généralisée. FOUCAULT, Une révolte à mains nues [1978, 2001 : 702].

Voici la dimension active d'un gouvernement : le monopole habilité de la force. ${ }^{44}$ Voyons ce qui se passe par rapport à un gouvernement provisoire ou à une Résistance organisée. Considérons pour l'instant comme indifférentes les finalités de la transition: qu'elles soient révolutionnaires ou bien constitutionnelles.

Un glissement de sens intéressant vient à ce propos du Kelsen de Principles of International Law de $1952 .^{45}$

Voici des extraits et des formules qui nous aideront pour la discussion.

a) La force est un monopole de la communauté. ${ }^{46}$

b) L'ordre social réserve l'emploi de la force uniquement à la communauté. ${ }^{47}$

\footnotetext{
${ }^{44}$ L'aspect à débattre est justement l'habilitation, plutôt que l'évidence de cette centralisation étatique (mieux : gouvernementale) de la force. D'où vient l'habilitation, et quelle est son ampleur ?

${ }^{45}$ KeLSEN, Principles of International Law [1952, 1959, 2003]. Le ton didactique de l'ouvrage kelsénien nous permet de mieux saisir les intentions définitoires de l'auteur.

${ }^{46}$ KELSEN, "The force monopoly of the Community", dans Principles of International law [1952, 2003 : 13]. Tirons du texte en anglais un extrait très riche, en emphatisant par le cursif des expressions qui, pour notre recherche, sont particulièrement significatives : «In stipulating sanctions, the legal order authorizes a definite individual to perform the coercitive act constituting the sanction. This individual, in carrying out the sanction, may be considered to act as an organ - centralized or decentralized - of the community constituted by the legal order. Hence the sanction may be considered to be an action of the community. That means that the act performed by an individual is imputed to the community » [Ibidem].
}

Dès maintenant soulignons, donc, que l'individu (qui pourrait être un non-citoyen) agit en tant qu'organe de la communauté, en appliquant, mieux : en «portant jusqu'au bout », en « exécutant » (carrying out) la sanction, c'est-àdire la norme. Relevons ensuite que, lorsqu'un individu applique la sanction, la norme qui préside (et préexiste) à cette dernière est rendue efficace au nom de la communauté (elle est «imputée » à la communauté, et non pas à l'individu). Soulignons encore que, la communauté étant constituée par l'ordre juridique, l'individu aussi fait partie de cet ordre. C'est donc par l'intermédiation de la communauté que l'individu est relié à l'ordre juridique, et non pas par l'intermédiation de l'ordre juridique que l'individu est relié à (fait part de, agit au nom de) la communauté. En d'autres termes, il/elle est un organe de la communauté (qui est évidemment constituée par l'ordre juridique), et non pas de l'ordre juridique. Si la reconstruction que nous proposons (tirée, pour une partie, de BoBBIO, Law and force [1965], Diritto e forza [1966] qui en est une traduction révisée, Hans Kelsen [1973], et Dalla struttura alla funzione [1977]) est correcte, comme nous le souhaitons, Kelsen devrait introduire ici un glissement, tantôt explicite tantôt déductible, du concept d'ordre juridique vers celui d'organisation.

${ }^{47}$ KELSEN, "The force monopoly of the Community", dans Principles of International law [1952, 2003 : 14]. Tirons encore de l'original (nous soulignons). «If a social order provides that coercive acts shall be performed only under definite conditions, determined by it, and only by definite individuals, likewise determined by it, and if we consider these individuals as organs of the community constituted by the social order, we may say that the social order 
Ce qui nous intéresse ici est évidemment le concept de « communauté » (serait-elle une entité nationale ou une organisation ?) et ce qu'agir au nom de la communauté signifie et implique.

Pour le cas d'une Révolution la question est nette : les révolutionnaires agissent au nom d'une communauté qui n'existe pas (du moins, pas encore). Une stratégie constitutionnelle s'accompagne de la pratique révolutionnaire, car la communauté de l'avenir a besoin d'une légitimation (qui se concrétisera ex post facto, on le présume et on le souhaite). Une transition qui trouve son origine dans une révolution (ou bien dans une rupture révolutionnaire) débouche sur une constitution. ${ }^{48}$

Dans le cas des Résistances les enjeux sont plus compliqués, car la communauté existe (si elle n'existait pas, parler de « résistance " n'aurait aucun sens) et la transition n'est pas aboutie au moment de l'écriture constitutionnelle. Pour l'énoncer dans des termes plutôt conventionnels, la tension entre faits (la Résistance) et normes (la Constitution) n'est pas « réglée » par la loi !

Au sein même de la catégorie de Résistance on dépiste une tension pareille. Les faits de la Résistance ne sont pas susceptibles de devenir forcément les normes de la transition « résistante ». ${ }^{49}$

De surcroît, sur le plan historique, ce passage de la Résistance comme fait à la Résistance comme norme juridique-constitutionnelle ne s'est produit que rarement et occasionnellement.

Il y a deux conséquences encore à tirer de la vision kelsenienne. D'abord, si la force est monopole de la communauté, cela implique que la violence, une fois monopolisée par la communauté, devient force légitime. Et, si la violence est force, cette dernière est violente ! Walter Benjamin dans son Zur Kritik der Gewalt (1921) a consacré à cette dialectique «stupide » et «baroque » des pages qui se révèlent, encore aujourd'hui, inépuisées. ${ }^{50}$

Il en découle que tout monopole de la force est violent. Il est donc soit révolutionnaire, soit résistant, soit insurrectionnel, soit rebelle, soit terroriste, soit avant-gardiste, soit partisan, etc. Tout ordre étatique, de type constitutionnel donc, est intimement et par définition du moins éversif !

Ensuite, si on peut agir la communauté (à savoir : agir au nom de la communauté), elle aussi est de nature transitoire, car elle peut être transformée par ceux qui agissent en son nom.

Résumons : la transition est violente alors que la constitution est forte, mais la communauté est surtout transitoire.

Le caractère transitoire, associé à l'exercice de la violence, fait que la communauté est habitée par les éversives, les terroristes, les rebelles, les insurgés, les révolutionnaires, les clandestins, les résistants.

reserves the employment of force to the community. Such a social order establishes a force monopoly of the community $»$.

Les interprétations qui portent sur le présumé caractère réaliste de cette (éclectique ?) sortie kelsénienne sont bien connues, et parfois elles centrent un point de «vulnérabilité » (mieux : d'ouverture) du normativisme de Kelsen (qui reste pourtant inassimilable, par exemple, à Olivecrona ou à Ross, pour n'en citer que les deux assonances au law as force, sinon as violence !).

Parmi bien d'autres, voir RAZ, The concept of a legal system [1970, $1980:$ ex. 78], The authority of law [1979, 2009 : ex. 122, 293] ; CowAN, Law without force [1971]. Le développement réaliste de la vision kelsénienne, jusqu'à en reprendre la formule normative de «ought to », se retrouve chez l'oxonien John Lucas (LuCAS, The principles of Politics, [1966: 16]).

${ }^{48}$ Il s'agit de la vision « en trois temps » que le continuisme classique nous a léguée, une vision largement majoritaire en politologie et presque unanime en théorie du droit. Notre travail de recherche la conteste et la démantèle.

${ }^{49}$ La dialectique Résistance / Terreur, souvent dramatiquement évoquée à ce sujet, le confirme.

${ }^{50}$ Une tentative d'application de la vision benjaminienne à la violence révolutionnaire, sous l'angle de la déconstruction et avec des insertions lacaniennes, mais qui ne tient pas compte ni de Kelsen, et de la critique à la tradition kantienne que le juriste proposait, ni de Weber, et de l'élargissement de la critique marxienne au pouvoir charismatique, tout en s'adressant à une problématique aussi que bizarre « lecture adornienne faite par Žižek » (sic !), est présente dans le volume très intéressant de MAGUn, La Révolution négative. Déconstruction du sujet politique [2009]. 


\section{4 ÉVERSIFS, TERRORISTES, REBELLES, INSURGES}

La Nation interroge. La Nation s'inquiète. La Nation juge. A toutes ces questions angoissées, nous répondons: Ce que nous sommes? Nous sommes les rebelles. ViannaY, Nous sommes les rebelles [1946: 11].

Omettons pour l'instant de prendre en considération la catégorie générale de « révolutionnaire ${ }^{51}$, qui est une sorte de catégorie générale dont les autres découlent à titre de spécification «fonctionnelle» (par exemple les «terroristes» qui auraient pour but la déstabilisation de l'ordre politique, et donc se situeront dans une articulation uniquement fonctionnelle, et surtout éventuelle, de la Révolution, ou les « éversifs » qui se concentreraient sur la sortie de l'ordre constitutionnel, plus ou moins violente, et surtout pas forcément considéré comme telle); ou en raison des étapes du processus révolutionnaire, de la scansion «temporelle » de la prise du pouvoir (par exemple les « rebelles », qui caractérisent en principe une toute première phase de la Révolution, ou les «insurgés » qui en caractérisent les phases les plus mûres).

En l'absence d'un processus révolutionnaire abouti ${ }^{52}$ - et c'est le cas notamment de la France et de 1'Italie au lendemain de la libération nationale ${ }^{53}$ - lesquelles, parmi les catégories considérées, correspondraient à la phase de la transition caractérisée par la Résistance ? Lesquels, parmi les éversifs, les terroristes, les rebelles et les insurgés, pourraient correspondre à la définition de « résistants »?

Prévenons tout d'abord que le droit constitutionnel général ne contemple pas de telles catégories, alors que le droit international les légitime toutes, sur la base des constatations souvent substantialistes (ex. la résistance contre une dictature particulièrement oppressive et violente peut arriver jusqu'aux actes de terrorismes, lorsqu'ils sont dirigés uniquement contre l'armée ou contre le dictateur, et, en tout cas, ne touchent pas à la population civile).

Le droit pénal, auquel nous devons l'analyse des cas qui nous concernent davantage. Tels sont, par exemple, pour ne parler que de l'Italie au lendemain de l'armistice,

$51 \mathrm{Au}$ sujet des « révolutionnaires » on suit la problématisation proposée par HoBSBAWM, Revolutionaries [1974, 2001].

${ }^{52}$ Considérons ici et le concept de processus révolutionnaire souvent analysé par les historiens (donc même là où il se produit une certaine « continuité » avec l'ordre étatique et constitutionnel précédent, tout comme dans le cas de la révolution fasciste, qui en fait s'est autoproclamée comme telle), et le concept juridique de révolution qui, d'un chef est sensiblement limité dans sa portée (en se limitant souvent à la « simple » relève de la rupture de l'ordre constitutionnel), en étant pourtant, de l'autre, bien plus complexe dans sa détection en raison de la méthode « rétrospective » que l'on doit majoritairement appliquer.

53 Au lendemain de la libération nationale (1944 pour les Français et 1945 pour les Italiens) on ne peut pas parler encore d'une révolution, soit-elle politique ou juridique. En fait, d'une façon qui est bizarre encore aujourd'hui, les historiens n'ont jamais étiqueté la libération italienne et française par le terme de « Révolution ». Elle le fut, et comme rupture constitutionnelle (nous le verrons lorsqu'on contestera la thèse de l'inexistence juridique de l'ordre de Vichy, ainsi que la thèse de la continuité du règne d'Italie) et comme instauration d'un nouvel Etat, une nouvelle constitution, un nouvel ordre politique, une nouvelle forme d'Etat (au moins dans le cas italien) et de gouvernement. Pour l'instant, toutefois, contentons-nous d'accepter une position de compromis : au lendemain de la libération du territoire national, et dans l'atteinte de la convocation ou de l'élection d'une assemblée constituante, ainsi que dans les nombreux cas de prorogatio des anciens pouvoirs légitimes (de droit ou de facto), on ne parlera pas encore de « Révolution ». 
a) l'attentat de Via Rasella, à Rome, du 23 mars 1944, commis par le GAP roman ${ }^{54}$, sous les directives de la junte militaire du CLN, ou

b) la présumée rétorsion à l'attentat partisan de Via Rasella, dans les faits : le massacre de 335 prisonniers des fascistes et des nazis, du 24 mars 1944 près des Fosse Ardeatine, à Rome, commis sous la responsabilité directe et ultime, outre des exécuteurs matériaux, des chefs allemands Herbert Kappler et Erich Priebke, les capitaines Kochler et schutz du général Maeltzer, du maréchal Kesselring ${ }^{55}$;

c) les attentats et les exécutions sommaires du «triangle de la mort » commis par les partisans de la Volante Rossa après la libération italienne de $1945^{56}$, mais en continuité substancielle avec la Résistance et avec la guerre de libération ${ }^{57}$, ainsi que

d) les représailles des nazis, des fascistes, des Résistants, qui ont caractérisé la période suivant la «proclamation» de la République sociale italienne, Etat national républicain, du 23 septembre 1943, et qui ont intéressé uniquement son territoire. ${ }^{58}$

Analysons plus de près les arguments en droit pénal et en droit constitutionnel dans le cas ci-dessus, qui nous aideront pour des repères fondamentaux aux fins de cette recherche.

\subsection{Banditen! L'attentat de Via Rasella du 23 mars 1944}

La décision du Tribunal militaire de Rome du $1^{\mathrm{er}}$ août 1996 se révèle intéressante pour nous, sous plusieurs aspects. Préalablement le juge offre une qualification très nette de l'attentat, qui fut un « acte de guerre reconductible à l'Etat italien », quoique commis par des sujets qui, aux sens du droit international, «n’étaient pas des belligérants légitimes » :

Esso fu un vero e proprio atto di guerra riferibile allo Stato italiano anche se commesso da persone che, secondo il diritto internazionale, non erano legittimi belligeranti. Il collegio condivide pienamente e senza riserve le argomentazioni già espresse dalla sentenza "Kappler", ribadite in maniera definitiva ed incontestabile, per l'autorevolezza della decisione, dalla pronuncia delle Sezioni Unite Civili della Corte Suprema di Cassazione (sentenza 9/5/1957).

${ }^{54}$ Pour une analyse, voir MAGLIACANE - RUBINO, Forme e crisi della norma-stato [2009 : chap. 4].

${ }^{55}$ Pour une analyse, voir MAGLIACANE - RUBINO, Forme e crisi della norma-stato [2009 : chap. 4].

56 Pour une étude historique approfondie, voir RECCHIONI, Ultimi fuochi di Resistenza [2009] et Il tenente Alvaro, la Volante Rossa e i Rifugiati politici italiani in Cecoslovacchia [2011].

57 Voir DONDI, La lunga liberazione [1999, 2004].

${ }^{58} \mathrm{Ce}$ qui, par exemple, nierait toute légitimation, même uniquement sur le plan formel, aux « représailles » allemandes des Fosse Ardeatine, à Rome, en considérant comme victimes de l'attentat de Via Rasella des hommes enrôlés dans la police politique du Reich et appartenant à une division territoriale qui, après la proclamation de la RSI, permanait toujours directement sous le contrôle du Reich (c'est-à-dire Bozen, qui ne faisait pas partie de la RSI). La rétorsion nazie, donc, n'était pas légitimée, même pas en tant qu'acte de guerre, au contraire de ce qu'ont déclaré le Tribunal et la Cour de Cassation, on le verra, qui ont jugé coupables les responsables de l'opération de rétorsion, Herbert Kappler et le SS-Hauptsturmführer Erich Priebke uniquement pour les morts « en plus » qu'ils n'étaient pas autorisés à exécuter. En fait, la rétorsion a été un acte de terrorisme (ce qui est, par ailleurs, conforme à l'analyse purement politique des faits). Selon le Tribunal militaire de Rome, « la logique pervertie de la rétorsion se concrétise dans une vie de fait vivant à terroriser la population et décourager d'autres attentats ou sabotages » (Trib. Mil. Rome, décision du 1 août 1996). 
Ensuite, la cour semble dépister ou au moins induire l'existence d'une sorte de précondition de la «guérilla » partisane (qui serait articulée évidemment même dans des actes de guerre peu « réguliers ») dans l'occupation allemande du territoire national italien.

L'attentato non fu ispirato da finalità personali ma da quella di compiere un atto ostile verso le FF.AA. della Germania, che era in guerra con l'Italia dall'ottobre 1943 ed aveva occupato militarmente gran parte del territorio nazionale.

Non può dubitarsi che esso fosse un atto di guerra dal momento che il legittimo governo italiano aveva incitato coloro che si trovavano nelle zone occupate a ribellarsi alle forze nemiche ed a compiere tutti i possibili atti di sabotaggio e di ostilità per contribuire alla liberazione, così come facevano le forze armate regolari a fianco delle Nazioni Unite.

Les actes de terrorisme seraient, donc, selon l'avis du juge militaire (et il est fort important qu'il s'agisse d'un tel type de juridiction), des actes de guerre, ouvertement légitimes, et de plus justifiés même sur le plan «éthique » en raison de l'occupation militaire.

Ce qui est particulièrement important dans cette reconstruction judiciaire est, d'ailleurs, la thèse que tous les actes de guerre (y compris « tous les actes de sabotage et d'hostilité ») seraient légitimes juridiquement lorsqu'ils sont commis sur «incitation à la rébellion » qui vienne du « gouvernement légitime ». 59

Or, quoique méritoire - surtout si l'on considère que la période des premières années '90 fut caractérisée par une sorte de « révisionnisme » historique sur la ligne des travaux plusieurs fois cités de Claudio Pavone ${ }^{60}$ et de Renzo De Felice ${ }^{61}$ visant à relire la Résistance sans en exclure la catégorie de «guerre civile » (et sans pourtant, il faut le dire, au moins chez Pavone et De Felice légitimer l'expérience de la République de Salò) - la thèse judiciaire est extrêmement faible là où elle se concentre sur le « gouvernement légitime » (question clairement non secondaire !) qui serait censé, en outre, être doté d'une capacité de transmission d'ordres et directives de façon supposée univoque et peu ou point équivoque.

\section{BIBLIOGRAPHIE}

ANDERSON, Perry [2015] - « The House of Zion », in new Left review, 96, nov-dec. 2015, 5.

ANDRIEU, Kora [2012] - La justice transitionnelle. De l'Afrique du Sud au Rwanda, Paris, Gallimard (Folio, Essais).

BASSO, Luca [2006] - Individuo e comunità nella filosofia politica di G. W. Leibniz, Rubbettino, Soveria Mannelli.

${ }^{59}$ En ce qui concerne strictement le droit pénal, le juge souligne que l'attentat n'était pas inspiré par des motivations personnelles. Ce principe est important, puisqu'il serait adopté, par comparaison inversée, contre Priebke, jugé au contraire, plus que violent, « terriblement sadique ».

${ }^{60}$ L'importance de PAVONE, Una guerra civile. Saggio storico sulla moralità nella Resistenza [1991, 1994, 2006, 2009] va certainement au-delà de l'analyse des trois guerres qui se dégagèrent en Italie après le 8 septembre : patriotique, de classe, et... civile.

${ }^{61}$ Ce n'est pas le seul volume publié posthume de sa remarquable histoire d'Italie sous l'angle visuel de Mussolini (DE FELICE, Mussolini l'alleato. II. La guerra civile [1997]) à être controversé politiquement, mais toute une série d'articles, de pamphlets, d'entretiens, de livres « provocateurs », venant d'ailleurs d'un ancien trockijste et ensuite dissident communiste de 1956, visant tous à re-établir une sorte de parité d'armes sinon une véritable équivalence morale entre la Résistance et la République de Salò. Voir, pour tous, DE FELICE, Il rosso e il nero [1995]. 
BIANCO, Dante Livio [1954, 2006] - Guerra partigiana, préfaction N. Bobbio, introduction N. Revelli, Torino, Einaudi.

BobBIo, Norberto [1977] - Dalla struttura alla funzione. Nuovi studi di teoria del diritto, Milano, Ed. Comunità.

Bоввіо, Norberto [1975] - Gramsci e la concezione della società civile, Milano, Feltrinelli.

BobBIO, Norberto [1973] - Hans Kelsen, in Riv. It. Fil. Dir., 1973, p. 425.

Bоввio, Norberto [1966] - Diritto e forza, in Riv. Dir. Civ., 1, 1966, p. 119 (traduction révisée de BOBвIO [1965])

BoввIO, Norberto [1965] - Law and force, in The Monist, 2, 1965, p. 321.

BobBIO, Norberto - RUSCONI, Gian Enrico [1992] - Lettere sull'azionismo, dans Il Mulino, novdéc. 1992, 1021-1029.

BocCA, Giorgio [1995, 2012] - Storia dell'Italia partigiana. Settembre 1943 - Maggio 1945, con introduzione di M. Revelli, Milano, Mondadori.

BocCA, Giorgio [1945, 2004] - Partigiani della montagna, Milano, Feltrinelli.

Borella, François [1960] - L'évolution de la Communauté en 1960. De la Communauté constitutionnelle à la Communauté conventionnelle, « Annuaire français de droit international », $6,1960,1,925$.

BORELLA, François [1958] - L'évolution politique et juridique de l'Union française depuis 1946, Paris, LGDJ.

CARTIER, Emmanuel [2005] - La transition constitutionnelle en France (1940-1045). La reconstruction révolutionnaire d'un ordre juridique 'républicain', Paris, LGDJ.

Chaliand, Gérard [1970] - La Résistance palestinienne, Paris, Seuil.

Chomsky, Noam [2000] - Rogue States. Crisis in Balkans and East Timor Retrospective, Cambridge, South End Press.

Chomsky, Noam - Clarke, Ramsey - SAÏD, Edward [1999, 2002] - La loi du plus fort. Mis au pas des Etats voyoux, La Madeleine de Nonancourt, Le Serpent à Plumes.

Courtois, Stéphane - Cowan, Thomas H. [1971] - Law without force, “California L. R.”, 1971, p. 683.

DARdot, Pierre - LAVAL, Christian [2015] - Commun. Essai sur la Révolution au XXI siècle, Paris, La Découverte. 
De Felice, Renzo [1997, 1998] - Mussolini l'alleato. II. La guerra civile (1943 - 1945), Torino, Einaudi.

De FeliCE, Renzo [1995] - Il rosso e il nero, Milano Baldini \& Castoldi.

DoNDI, Mirco [2004] - La lunga liberazione. Giustizia e violenza nel dopoguerra italiano, Roma, Editori Riuniti.

DREYFus, François-Georges [1996] - Histoire de la Résistance 1940-1945, Paris, Editions de Fallois.

ENGLAND, Andrew [2015] - «Barriers to entry », in Financial Times, 26 nov. 2015, 5.

FouCAult, Michel [1978, 2001] - Dits et écrits II, 1976-1988, Paris, Gallimard.

FreIXES, Teresa [2018] - 155 : Los días que estremecieron a Cataluña, Madrid, Editorial Doña Tecla.

GrAmSCI, Antonio [1978] - Americanismo e Fordismo. Quaderno 22, Torino, Einaudi.

Gresh, Alain [2010] - De quoi la Palestine est-elle le nom ?, Arles, Babel - Actes Sud.

GRESH, Alain [1983] - Olp. Histoire et stratégies. Vers l'Etat palestinien, Paris, Spag-Papyrus.

GuARINO, Giuseppe [2004] - Personalità giuridica di diritto internazionale : il caso dell'Organizzazione per la Liberazione della Palestina, dans AA. VV., Studi di diritto internazionale in onore di Gaetano Arangio-Ruiz, Napoli, Novene, vol. I., p. 85.

HeCHICHE, Abdelwahab [1973] - Renaissance et déclin de la Résistance palestinienne, « Politique étrangère », 38, 1973, 5, 597.

HEYDE, Veronika [2010] - De l'esprit de la Résistance jusqu'à l'idée de l'Europe. Projets européens et américains pour l'Europe de l'après-guerre (1940-1950), Bruxelles, Peter Lang (Euroclio n.52).

HobsBAwM, Eric J. [1974, 2001] - Revolutionaries, New York, The New Press.

HobsBawm, Eric J. [1994] - The Age of extreme. The short Twentieh century 1914-1991, London, Michael Joseph.

KELSEN, Hans [1952, 2003] - Principles of International Law, Clark (NJ), Lawbook Exchange.

LÉGASSIK, Martin [1998] - Myth and Reality in the Struggle against Apartheid, "Journal of Southern African Studies", 24, juin 1998, 2, 3. 
LEGNANI, Massimo - VENDRAMINI, Ferrucio [1990] - Guerra, guerra di liberazione, guerra civile, Milano, Franco Angeli.

LuCAS, John R. [1966] - The principles of Politics, Oxford, Clarendon Press.

Magliacane, Alessia J. [2019] - L'uomo Mosè e il moroteismo. Alle radici della postdemocrazia italiana, Florence - Paris, Classi .

Magliacane, Alessia J. [2017] - Rupture de l'Histoire, continuité de la Résistance. Une introduction méthodologique, Florence - Paris, Classi.

Magliacane, Alessia J. [2016] - Le temps non linéaire de la Résistance. A l'épreuve de l'histoire, Florence - Paris, Classi.

Magliacane, Alessia J. [2015] - La Totalité contre la Résistance, Florence - Paris, Classi.

Magliacane, Alessia J. - Rubino, Francesco [2015] - Forme e crisi della norma-stato. Contributi per una critica del diritto, Florence - Paris, Classi.

Magun, Artémy [2009] - La Révolution négative. Déconstruction du sujet politique, Paris, L'harmattan.

MANDELA, Nelson [1995] - Un long chemin vers la liberté, Paris, Fayard.

MiCHEL, Henri - MiRKINE-GuETZÉVITCH, Boris [1954] - Les idées politiques et sociales de la Résistance. Documents clandestins 1940-1944, textes choisis et recueillis par H. Michel et B. Mirkine-Guetzévitch, préface de L. Fèvre, Paris, Puf (Esprit de la Résistance).

MongIN, Olivier [1995] - Vers la troisième ville, Paris, Hachette.

NEGRI, Antonio [1992] - Il potere costituente. Saggio sulle alternative del moderno, Milano, SugarCo.

PACINI, Giacomo [2014] - Le altre Gladio. La lotta segreta anticomunista in Italia. 1943-1991, Torino, Einaudi.

PAQUOT, Thierry [1997] - «L'invention du citadin », in Sciences humaines, n 70, mars 1997.

Pavone, Claudio [1991, 2009] - Una guerra civile. Saggio storico sulla moralità nella Resistenza, Torino, Boringhieri.

PAVONE, Claudio [1994] - La seconda guerra mondiale: una guerra civile europea?, dans RANZATO (dir.), Guerre fratricide. Le guerre civili in età contemporanea, Torino, Bollati Boringhieri.

PORTEILlA, Raphaël [1999] - Le nouvel Etat sud-africain. Des Bantoustans aux provinces, Paris, L'Harmattan. 
RANZATO (dir.), Guerre fratricide. Le guerre civili in età contemporanea, Torino, Bollati Boringhieri.

RAZ, Joseph [1979, 2009] - The authority of law. Essays on law and morality, Oxford - New York, Oxford Univ. Pr.

RAZ, Joseph [1970, 1980] - The concept of a legal system. An introduction to the theory of legal system, Oxford, Clarendon Press.

RECCHIONI, Massimo [2013] - Francesco Moranino, il comandante “Gemisto”. Un processo alla Resistenza, Roma, Derive\&Approdi.

RECCHIONI, Massimo [2011] - Il tenente Alvaro, la Volante Rossa e i rifugiati politici in Cecoslovacchia, Roma, Derive\&Approdi.

RECCHIONI, Massimo [2009] - Ultimi fuochi di Resistenza. Storia di un combattente della Volante rossa, Roma, Derive\&Approdi.

RÉMY, Jean [1985] - La ville dans la problématique wébérienne, dans Figures de la ville : autour de Max Weber, Paris, Aubier.

RILEY, Peter [2002] - Leibniz and the idea of the common good, dans Andreu - Echevaria Roldan [dir. 2002] - Ciencia, tecnologia y bien común. La actualidad de Leibniz, Valencia, UPV, p. 355.

SAÏD, Edward [2004] - D'Oslo à l'Irak, Paris, Fayard.

SAÏD, Edward [2003, 2004] - Culture et Résistance. Entretiens avec David Bersamian, Paris, Fayard.

SAÏD, Edward [1978, 2003] - L'Orientalisme. L'Orient crée par l'Occident, préface : T.

Todorov, Paris, Seuil.

SAlVADORI, Massimo L. [1972] - Gramsci e il problema storico della democrazia, Torino, Einaudi.

SCAMARDELLA, Antonello [2017] - Verità e paura, Firenze - Parigi, Classi.

Scoppola, Pietro [1995] - 25 aprile: liberazione, Torino, Einaudi.

TROCKIJ, Lev (TROTSKY, Léon) [1929, 1995] - Histoire de la révolution russe. 1. La révolution de février, traduction : M. Parijanine, introduction : J.-J. Marie, avant-propos : A. Rosmer, Paris, Seuil (Essais). 